\title{
Philosophiques
}

\section{Heidegger sur le chemin du langage}

\section{Peter McCormick}

Volume 1, numéro 2, octobre 1974

URI : https://id.erudit.org/iderudit/203011ar

DOI : https://doi.org/10.7202/203011ar

Aller au sommaire du numéro

\section{Éditeur(s)}

Société de philosophie du Québec

ISSN

0316-2923 (imprimé)

1492-1391 (numérique)

Découvrir la revue

Citer cet article

McCormick, P. (1974). Heidegger sur le chemin du langage. Philosophiques, 1(2), 15-36. https://doi.org/10.7202/203011ar

\section{Résumé de l'article}

La méditation la plus importante d'Heidegger sur le langage s'intitule « Le chemin vers le langage "; c'est le dernier chapitre d'Unterwegs zur Sprache. Si nous considérons la possibilité que le dernier Heidegger a quelque chose à apporter aux problèmes philosophiques du langage, nous devons déterminer exactement ce que Heidegger pense dans cette méditation. C'est pourquoi ma communication se bornera à une lecture détaillée et très restreinte de ce texte. La tâche plus intéressante mais, compte tenu de l'état des études sur Heidegger. moins prioritaire, c'est-à-dire l'évaluation des propos heideggeriens, et leur incorporation dans une optique plus large sur la philosophie du langage, m'occupera à une autre occasion. Je prendrai comme points d'appui l'introduction prolongée de la méditation heideggerienne et sa conclusion. Dans un troisième et plus court moment, je mettrai ensemble les résultats de ces deux premières analyses pour faire le point sur les éléments de base du dernier Heidegger sur le langage. Partout, mon intention serait d'ouvrir la possibilité d'une discussion dont les éléments essentiels seraient en fin délimités. 


\title{
HEIDEGGER SUR LE CHEMIN DU LANGAGE *
}

\author{
par Peter McCormick
}

Der Weg zur Sprache constitue la méditation la plus élaborée de Heidegger sur le langage dans Unterwegs zur Sprache.

Outre une introduction et une conclusion, cette méditation comprend trois parties principales. Je voudrais avant toute chose examiner l'intention de Heidegger dans cette méditation, telle qu'on la trouve exprimée dans l'introduction et la conclusion. Ces deux sections contiennent des déclarations importantes qui, comprises dans leurs grandes lignes, guideront notre accès à l'intelligence des parties principales de l'essai. Nous serions alors en ćtat d'estimer si cette intelligence est immédiatement accessible ou si elle requiert une lecture approfondie des méditations précédentes de Unterwegs.

1. Heidegger commence et termine son essai par la même citation de Novalis: «Ce que nul ne connaît, c'est justement le propre (Eigentümlichkeit) du langage, qu'il ne se préoccupe (sich bekümmert) que de lui-même » ${ }^{(211)}$. Ce texte, pense Heidegger, désigne ce qu'il appelle «le secret du langage ». Selon sa formulation, ce secret consiste dans le fait que le langage parle «... seul et solitairement avec lui-même ». Une telle formulation suggère l'idée d'une sorte de cercle linguistique. Pour le dire simplement, discuter la nature du langage, c'est nécessairement user du langage. Le point de vue adopté ici sur ce problème familier est inusité, puisque la formulation de Heidegger ne souligne pas comment il faut se servir du langage pour discuter du langage,

\footnotetext{
* Texte revu par Paul Ricoeur. Une ébauche plus courte de ce texte a été prononcée au 42ième Congrès de l'ACFAS à Québec le 9 mai sous le titre «Le dernier Heidegger et le langage $\gg$.
} 
mais que le langage est cela même que nous voulons discuter. L'accent principal est mis sur le langage et non sur le philosophe, plus précisément sur ce que Heidegger, suivant en cela Novalis, appelle la spécificité ou le propre du langage. Mais le fait important est la suggestion de Heidegger que cette spécificité enveloppe un mouvement circulaire. Le langage ne s'atteste que dans le parler des hommes et pourtant c'est le langage lui-même qui parle et qui parle «... seul et solitairement avec lui-même». Comment peut-on dire que le langage parle? Cette aporie est le point de départ de Heidegger.

Son intention est moins claire: « Nous voudrions faire l'expérience du caractère et de la portée (Character und Reichweite) de ce mouvement à partir du langage lui-même, en nous engageant dans l'entrelacs (Geflecht) ${ }^{(243)}$. Heidegger fait ici allusion au cercle línguistique, mais en un sens différent de la suggestion antérieure. Dans le cas présent, le cercle du langage se rapporte à ce que Heidegger se représente comme une relation en boucle (zurücklaufende), à l'intérieur du langage lui-même, entre ce qui, d'une part, resserre le langage et ce qui le délie. Les mots employés ici suggèrent que le langage consiste en un tissu, une texture, un entrelacs (Band). Cet entrelacs, tout à la fois, fait tenir ensemble la texture du langage et en maintient à part la texture. Il y a un mouvement dans le langage dans la mesure où ce qui fait tenir ensemble la texture du langage se replie sur soimême pour devenir ce qui maintient cette texture à part. Bien plus, cette courbure ne constitue pas seulement un mouvement, mais pour autant qu'elle forme boucle sur elle-même, elle donne au mouvement la forme d'un cercle. L'intention de Heidegger, en s'engageant ainsi dans cette texture, est de faire l'épreuve de son mouvement. Tout indique que Heidegger vise à éprouver la relation circulaire et dynamique entre ce qui unifie le parler du langage et ce qui différencie ce parler. Toutefois, une telle lecture pourrait s'avérer superficielle. Contentons-nous de noter en ce point que ce qui est dit ici de l'intention de Heidegger reste vague parce que nous-même sommes, pour l'instant du moins, en pleine confusion quant à ce qui est ici décrit comme la texture du langage, le mouvement du langage et l'expérience relative à ce mouvement. 
Un certain nombre de confusions se dissipent dès que Heidegger décrit comment il entend remplir sa tâche. Son programme est de suivre les indications de ce qu'il appelle «la formule du langage, en tant que langage, au langage ${ }^{(242)}$. Les italiques indiquent l'importance de la formule. Ce qui requiert ici une explication, ce n'est pas seulement le sens de la formule, mais sa présentation comme la formule d'un itinéraire. Le sens s'éclaire quelque peu si nous retournons aux remarques méthodologiques qui suivent immédiatement la citation de Novalis. Heidegger y pose que sa réflexion ultérieure ne gagne rien à être entendue comme une série de propositions sur le langage, car ces propositions ne reposeraient sur aucune preuve ni sur aucune démonstration. Ce qui suit doit plutôt être pris comme une série de conjectures. Heidegger suggère que nous renonçions à donner des démonstrations à ces conjectures sur le langage. Il nous faut plutôt «... faire l'expérience du chemin vers le langage à partir de ce qui se découvre chemin faisant (was sich ... unterwegs begibt) ...» ${ }^{(241)}$ Ainsi la notion de chemin est introduite sans autre explication, comme si elle était tenue pour familière en raison de son emploi fréquent dans les chapitres précédents. Sans les secours de ces usages antérieurs, on peut être amené à se représenter le langage comme quelque chose qui serait situé dans une région. Un chemin conduit à cette région. En suivant ce langage on est conduit là où la nature du langage, sa spécificité, se situe. Ce chemin toutefois demeure inconnu à tous ceux qui n'ont pas l'expérience du chemin et cette expérience n'est elle-même donnée qu'à ceux qui sont déjà sur le chemin du langage. Car être sur le chemin du langage est ce qui découvre le chemin du langage et le chemin du langage est ce qui nous permet d'accéder à la région où se situe la spécificité du langage.

Mais cette interprétation offre une difficulté immédiate, car il semble qu'il y ait ici cercle dans la mesure où le chemin du langage doit être éprouvé sur la voie du langage, tandis qu'en même témps la voie vers le langage doit suivre le chemin du langage. Ce cercle est en fait d'une ambiguïté centrale qui affecte l'usage heideggerien du terme $W e g$, terme qu'il considère aussi fondamental que ceux de logos, de $T_{a o}$ et de $I k i^{(198)}$. Le texte 
que nous discutons ici emploie le même mot de Weg pour ce que nous rendons ici par les deux mots de «chemin 》 et de «voie ». Une distinction s'impose ici entre un usage primaire du terme et l'un de ses sens appliqués. C'est le premier que j'ai rendu par le mot «chemin», tandis que le second a été rendu par le mot «voie»; nous dirons donc: suivre le chemin du langage devrait nous permettre de faire l'expérience de la voie vers le langage.

Mais ce qui reste obscur, c'est de savoir en quel sens nous pouvons parler de notre voie vers le langage, en quelque sens que ce soit, et plus précisément de savoir comment un tel chemin se découvre lorsque nous sommes sur la voie du langage. Cette obscurité résulte de l'insistance de Heidegger à user d'expressions métaphoriques pour décrire son entreprise, pratique qu'il ne s'emploie aucunement à discuter dans cet essai. Toutefois, il tire au clair quelques-unes des suggestions que ces expressions métaphoriques évoquent. Ainsi, se demande-t-il si un chemin vers le langage est nécessaire et même possible. En ce qui concerne la nécessité, Heidegger pose que, puisque l'aptitude de l'homme au langage est ce qui distingue particulièrement l'homme en tant qu'un homme, «...l'essence de l'homme repose (berubt) sur le langage " ${ }^{(241)}$; dès lors, un chemin vers le langage n'est pas nécessaire, puisque l'homme est déjà à la fois... dans le langage et auprès du langage ». En ce qui concerne la possibilité, Heidegger pose qu'un tel chemin est impossible pour autant que nous résidions déjà là où un tel chemin devrait conduire. Dès lors, la réponse à la première question, à savoir qu'un chemin vers le langage n'est pas nécessaire, semble impliquer la réponse à la seconde question, à savoir qu'un chemin vers le langage n'est pas possible.

Mais si l'on s'en tient pour l'instant à ces termes, quelle forme a l'argument en faveur de la première conclusion ? L'essence de l'homme est-elle reliée au langage, de telle façon qu'il faille conclure que l'homme est dans et auprès du langage (in der Sprache und bei der Sprache)? Heidegger parait bien ici introduire une confusion en s'appuyant sur ce qui n'est guère plus qu'un parallélisme verbal, lorsqu'il prétend que puisque l'essence de l'homme réside dans le langage, l'homme est déjà avec le 
langage, auprès du langage (bei der Sprache). Ce qu'il entend lorsqu'il dit que l'homme ou l'essence de l'homme est dans le langage reste extrêmement vague. Cette incertitude laisse plus d'un doute quant à la force de la première conclusion de Heidegger. Et puisque le second argument dépend du premier, le doute persiste quant à la force du second argument. Heidegger lui-même, il est vrai, exploite ce doute en refusant de répondre à sa seconde question par autre chose qu'une série de questions. Ce sont ces questions qui exigent, non seulement que l'on poursuivre la description de la métaphore du chemin, mais que l'on élabore plus avant l'intention de Heidegger dans cet essai.

Heidegger demande ici si l'homme se tient déjà là où cette essence semblerait le situer et où le chemin devrait le conduire, à savoir dans la région où la spécificité du langage est manifeste. Le chemin du langage est maintenant décrit du point de vue de sa largeur, comparée à celle des chemins qui mènent à d'autres régions. Toutefois, ce que Heidegger souligne; lorsqu'il dit: «...le chemin vers le langage en tant que le langage est-il le plus vaste que nous puissions penser? ${ }^{(242)}$, est un aspect de la métaphore du chemin qui se rapporte non aux différentes issues de différents chemins, mais à l'amplitude variable des chemins qu'il est permis de penser. Deux implications sont ici apparentes. La première est qu'il y a des chemins qui se laissent penser et des chemins qui ne se laissent pas penser. La seconde est que parmi les chemins qui se laissent penser, le chemin du langage, en tant précisément qu'il est langage, (sans doute le langage dans sa spécificité) est précisément le chemin le plus vaste qu'il soit permis de penser. Cette description, toutefois, paraît ajouter à la confusion puisqu'elle joint à l'ambiguïté délibérée du mot Weg l'ambiguïté additionnelle qui tient à la notion de largeur. Ce que nous avons ici à considérer, c'est une description métaphorique supplémentaire de ce que Heidegger tient pour l'accès à l'expérience du caractère spécifique du langage en tant que langage. L'obscurité qui s'ajoute aux termes employés pour élargir la métaphore vient de ce que les termes additionnels ont, comme ceux qui sont à la base de la métaphore du chemin, plus d'une signification, lors même qu'ils ne sont pas employés en connexion avec la métaphore du chemin. 
Outre cette description complémentaire de la métaphore du chemin, Heidegger suggère que «l'expérience évoquée dans la description de son projet parait fondamentalement une expérience pensante ». Cette expérience, semble-t-il, est en outre fonction de quelque sorte d'acte mental. Ainsi, quand Heidegger demande: "Sommes-nous dans le langage de telle façon que nous fassions l'expérience de son essence, que nous le pensions comme le langage, que nous percevions (vernehmen) ce qu'il a de propre (Eigene) en l'entendant? ${ }^{(242)}$, nous découvrons à la fois une opposition significative entre faire l'expérience de l'essence du langage et penser le langage comme langage, et la priorité de la perception du propre langage par rapport à cette pensée du langage. En termes plus ramassés, Heidegger semble proposer de penser l'essence du langage en tant que langage en tentant de percevoir attentivement le propre du langage. Toutefois, cette interprétation présente à son tour des difficultés. Qu'estce que Heidegger tente de décrire quand il parle de percevoir le propre du langage? Et quelle sorte d'activité représente : «... percevoir ce propre....»? Rien de cela n'est évident ni même expliqué; quand cette interprétation est placée côte à côte avec la première interprétation de Heidegger évoquée plus haut, la chose n'en devient pas plus claire. Ainsi, on pourrait présenter en ces termes l'interprétation complexe de l'intention de Heidegger :

a) faire l'expérience, à partir du langage lui-même, du caractère et de l'amplitude du mouvement refermé sur lui-même, de ce qui tout à la fois tient ensemble et tient à part la texture du langage, quand nous nous engageons nous-mêmes dans cette texture et,

b) penser l'essence du langage en tant que langage en tentant de percevoir avec attention le propre du langage.

Ce qui demeure obscur ici, c'est de savoir si «faire l'expérience » dans a), est équivalent à «penser » dans b), quelle relation existe entre l'essence du langage en tant que langage dans a) et l'ouverture et la fermeture de la texture du langage dans b) et quelle relation existe entre faire attention au propre du langage dans a) et s'engager dans la texture du langage dans b). 
2. Si la tentative d'expliquer pourquoi Heidegger appelle sa formule une formule d'itinéraire soulève tant de questions, la tentative d'expliquer la formule même de l'itinéraire en soulève plus encore. Dans la formule «porter le langage en tant que le langage au langage», Heidegger maintient que le terme de «langage » est employé dans un sens qui est chaque fois différent (Anderes) et pourtant le même (Selbe). L'identité, en chaque cas, est décrite par Heidegger comme «... ce qui fait tenir ensemble ce qui a été tenu à part (Auseinandergehaltene), à partir de l'unique chose sur quoi repose la spécificité du langage ${ }^{(242)}$. Si on suppose que l'unique chose dans laquelle réside la spécificité du langage peut être identifiée avec ce que Heidegger appelle à la fois «le langage en tant que le langage » et «l'essence du langage», tout indique alors que Heidegger implique que l'essence du langage comprend une relation entre deux aspects à la fois irréductibles (Anderes) et pourtant inséparables (Selbe). Quoi que ces aspects puissent être, Heidegger se borne ici à décrire leur relation plutôt que leur nature. Cette relation semble être de proximité puisque les deux aspects sont conçus comme, si l'on peut dire, rapprochés par une sorte de force gravitationnelle essentielle, tandis qu'ils sont tenus séparés par une sorte de force centrifuge essentielle.

Outre cette notion des aspects distincts de l'essence complexe du langage, pensés en termes d'identité et de différence, Heidegger dit que la formule du chemin annonce aussi «...l'entrelacs de relations dans lequel nous sommes déjà nous-mêmes impliqués » ${ }^{(242)}$. Heidegger pose que «le projet (vorbaben) d'un chemin vers le langage est entrelacé dans un parler...» ${ }^{(242)}$. Ce parler est le parler du langage et le parler du langage, continue Heidegger, «voudrait justement délivrer le langage et formuler cette représentation: ce qui atteste en même temps que le langage nous a nous-mêmes entrelacés dans le parler ...» ${ }^{(242)}$. Il ressort que Heidegger pose que le langage parle et que ce parler enveloppe l'intention de s'avancer sur le chemin du langage, parce que le langage est à la fois représentation et expression. Ainsi affirme-t-il que la proposition qu'on vient de citer $\ll .$. atteste en même temps que le langage nous a nousmêmes entrelacés dans le parler $»$. 
Mais il y a sûrement là une source de confusion. Nous serions tentés de dire en termes métaphoriques que le langage parle dans la mesure où, lorsque nous parlons, les mots que nous employons effectivement suggèrent d'eux-mêmes par association d'autres mots que nous pourrions employer à un autre moment. Venons-nous à faire usage de l'association suggérée par les expressions qui précèdent, nous pouvons alors songer à étendre notre métaphore initiale et dire que le parler du langage implique ou entrelace notre propre parler dans une sorte d'entrelacs verbal. Quel sens cela peut-il avoir de dire que le parler du langage implique notre chemin vers le langage? Même si on admet que le chemin vers le langage consiste à penser la relation entre l'essence de l'homme et l'essence du langage, comme Heidegger le fait, comme l'expérience attentive de cette relation, on ne comprend pas clairement en quel sens il est possible de dire que le parler du langage implique une telle pensée. Car quand nous disons que le parler comprend le penser, la confusion vient de ce que nous employons manifestement parler dans le contexte métaphorique de parler du langage, tandis qu'il n'est pas clair si penser est employé dans le même contexte ou dans quelqu'autre contexte. Quoi qu'il en soit, Heidegger continue à élaborer la notion d'entrelacs telle qu'il vient de l'introduire. Heidegger indentifie maintenant cet entrelacs avec la région (Bereich) qui nous est apparue impliquée par la métaphore du chemin menant à quelque zone où l'essence du langage est située. Heidegger s'emploie ici à combiner les métaphores séparées du chemin et de l'entrelacs alors qu'auparavant il avait combiné les aspects séparés de la même métaphore. La fonction de la formule du chemin est de nous inviter «non à écarter l'entrelacs mais à le délier pour permettre un regard (es gewahrt den Blick) jeté sur la libre co-appartenance (Zusammengebören) des relations nommées par la formule ${ }^{(243)}$. La métaphore de l'essence du langage comme un entrelacs suggère que les traits de cette essence sont, pour ainsi dire, maintenus en place par une sorte de lien et la tâche exigée par la pensée de l'essence du langage est de délier l'entrelacs suffisamment pour glisser un regard dans ce lien: "Peut-être cet entrelacs est-il traversé par un lien qui, d'une manière sans cesse surprenante, délie le langage en ce qu'il a de propre». Heidegger ajoute : «Il s'agit 
de faire l'expérience, dans l'entrelacs du langage, de ce lien qui délie » ${ }^{(243)}$.

Il est à nouveau difficile de dire exactement ce que Heidegger est en train de décrire. D'un côté, la notion du langage vu comme un entrelacs de relations paraît aller de soi ; également, l'hypothèse d'un principe linguistique qui rendrait compte du rapport variable qui règlerait les rapports de structure entre des langages aussi différents que l'allemand ou le japonais est assez familière. Mais la description de ce principe hypothétique comme une libre co-appartenance des relations d'identité et de différence régnant entre les trois emplois du mot «langage » dans la formule du chemin demeure obscure. Heidegger semble vouloir dire que l'interdépendance des aspects inséparables et irréductibles de l'essence composite du langage est ordonnée de manière à constituer le caractère spécifique du langage et que cette structure constititutive peut être pénétrée par le regard dans la mesure seulement où la structure superficielle du langage révèle pour un regard suffisamment pénétrant sa relation essentielle à sa structure profonde.

Mais ce sont là des expressions «transformationnelles 》 qui, comme telles, sont non seulement anachroniques, mais de valeur douteuse dans le cas présent, même si on les considère indépendamment de l'histoire récente de leur développement. Selon les propres termes de Heidegger, la relation entre le lien et l'entrelacs, dans ce modèle métaphorique de l'essence du langage, est celle d'une relation en boucle; comme telle, elle est comprise dans la théorie de l'information comme un cercle. C'est le caractère et l'amplitude du mouvement de cette relation circulaire, avons-nous dit, que Heidegger entreprend d'éprouver à partir du langage lui-même. Plus nous suivons avec persévérance ce que le chemin indique, nous dit Heidegger, "plus le langage se montre clairement lui-même en ce qu'il a de propre, plus le chemin vers le langage devient, chemin faisant, significatif pour lui-même et c'est d'autant plus décisivement que se transforme le sens de la formule du chemin. Elle perd son caractère de formule, elle est imprévisiblement un écho silencieux (lautlosen Einklang) qui nous laisse entendre un peu de ce qui fait le propre du langage ${ }^{(243)}$. 
3. Quelles sont donc les propositions principales où Heidegger exprime son intention, son mode d'approche, dans l'introduction à cette méditation centrale? On peut dégager des remarques de Heidegger un certain nombre de propositions distinctes dont chacune peut suggérer un certain nombre d'interprétation diverses. Eu égard à notre projet, dix propositions relativement distinctes requièrent, à mon avis, notre attention : deux traitent du point de départ de Heidegger, sept de ses remarques de méthode et une de son traitement du problème de l'évaluation. Comme ces propositions peuvent être dégagées des remarques introductives de Heidegger lui-même, nous pouvons y renvoyer sans inconvénient comme aux propositions I.

Puis la citation de Novalis, qui sert de point de départ de Heidegger suggère que ( $I, 1)$ le langage parle seul et solitairement avec lui-même, et que $(\mathrm{I}, 2)$ la spécificité du langage est inconnue. Les propositions $(1,1)$ et $(1,2)$ se réfèrent l'une à l'autre en ceci que Heidegger accepte l'assertion de Novalis selon laquelle $(I, 2)$ vaut parce que $(I, 1)$ vaut. La relation entre ces deux propositions initiales et les suivantes est plus difficile à déterminer. Lorsqu'il explique aussi bien l'énoncé de la formule qui validait sa réflexion sur le langage que le contenu de la formule elle-même, on peut estimer que Heidegger pose les sept propositions suivantes. (I, 3) Quoique la spécificité du langage soit inconnue, on peut néamoins la considérer en termes métaphysiques comme ce qui repose dans une région. (I, 4) Il existe un chemin vers cette région et ce chemin est accessible à tout être humain parce qu'il est lui-même tel en raison d'une relation qui existe entre l'essence de l'être humain et celle du langage. (I, 5) Quoique l'homme soit déjà sur la voie du langage en raison de cette relation essentielle, la relation entre l'essence d'être humain et celle de langage n'est pourtant pas telle que l'homme pense l'essence du langage spécifiquement comme langage. (I, 6) Penser la relation entre l'essence d'être humain et l'essence du langage comme le langage, c'est faire l'expérience du chemin vers le langage. (I, 7) Une telle pensée se produit lorsque l'homme, portant son attention sur le propre du langage dans le parler du langage, perçoit ce propre. (I, 8) L'essence du langage comprend une relation entre deux aspects inséparables 
quoiqu'irréductibles, à savoir ce qui unifie la différenciation et ce qui différencie l'unité de cet entrelacs. (I, 9) La relation entre le principe d'unité et le principe de différence au sein de l'essence du langage est d'une nature dynamique et circulaire du parler du langage. Finalement, Heidegger maintient ( $I, 10)$ que les énonciations sur le langage ne gagnent rien à être comprise comme des propositions démontrables, puisque ces énonciations valent seulement comme des conjectures pour lesquelles ni preuves ni démonstrations ne peuvent être avancées.

Maintenant, chacune de ces énonciations présente des difficultés propres dont quelques-unes au moins ne tiennent pas proprement aux formules originales de Heidegger dont elles ont été directement tirées ou indirectement dégagées par paraphrase. Il est manifeste en outre que l'enchaînement de ces énonciations reste elliptique et appelle une articulation plus soigneuse. Mais, en dépit de ces difficultés, ou d'autres que l'on n'aurait pas de peine à faire apparaître, cette série d'énonciations a au moins l'avantage de nous conduire au coeur de l'essai de Heidegger sous la conduite de plusieurs questions fondamentales. Ainsi, nous souhaiterions davantage d'éclaircissements sur le sens dans lequel on peut dire que le langage parle. En outre, on attendrait un développement plus clair sur le point de savoir comme Heidegger comprend l'essence du langage en tant que langage et jusqu'à quel point cette essence se prête à une description qui ne serait pas seulement métaphorique. Finalement, on aimerait trouver des indications concernant la conscience que Heidegger a pu prendre du problème des critères d'évaluation applicable à ses propres réflexions, concernant en outre toutes les autres suggestions du même ordre tendant à des procédures d'évaluation.

En dépit de ces formules-guides, la lecture répétée du texte principal de Heidegger lève une myriade de détails embarrassants; toute tentative pour organiser ces détails rend rapidement conscient de l'arbitraire inévitable de l'interprétation, et déjà de l'arbitraire de l'interprétation mise en jeu par la sélection et la formulation des dix énoncés initiaux dont les questionsguides sont tirées. 
Il semble dès lors raisonnable de mettre provisoirement entre parenthèses toute lecture cursive du texte de Heidegger et de se porter directement aux conclusions que l'essai atteint à son terme. Si dès le début nous réussissons à saisir non seulement ce que Heidegger se propose d'accomplir dans son essai, mais encore ce qu'il pense avoir accompli à son terme, nous serons peut-être en état de suivre plus fidèlement le cours de ses remarques dans. le corps du texte. Je propose donc de considérer la fin de l'essai. de Heidegger et de tenter provisoirement d'isoler ses conclusións.

4. L'essai de Heidegger s'achève sur la répétition du point de départ, la citation de Novalis. Heidegger remarque que Novalis comprend la spécificité inconnue du langage comme ce qui permet de reconnaitre le langage pour ce qu'il est. Puis il cerne cette spécificité (Eigentümlichkeit) de l'essence du langage en tant que langage en la déterminant dans des termes quelque peu emphatiques: «...la proximité du rendre propre et du faire advenir» (die Nàhe Eignens und Ereignens) ${ }^{(265)}$. La spécificité du langage accède à cette proximité, dit Heidegger, "par l'expérience de l'être du langage en tant que dire (Sage), dire dont le montrer (Zeigen) repose dans l'avènement (Ereignis... ${ }^{(265)}$. Ainsi Heidegger pose que le caractère propre de la spécificité de l'essence du langage en tant que langage est déterminé par ce qu'il appelle l'avènement. Au sein de l'avènement repose la manifestation de l'essence linguistique comme dire. Le caractère propre de cette spécificité est toutefois déterminé primitivement par la proximité du rendre propre et du faire advenir. Heidegger dit explicitement que ce n'est pas ici le lieu de réfléchir sur cette notion de détermination primitive, si bien que la question reste sans réponse de savoir ce que pourrait bien être la relation entre la détermination du caractère propre de l'essence linguistique en tant que dire et la détermination primitive de ce caractère. Cette question spéciale toutefois ne doit pas nous arrêter pour l'instant puisque, à la différence des questions développées dans la lecture de l'introduction, les questions qui demeurent ici en suspens se posent en conclusion d'une analyse développée que nous n'avons pas encore prise en considération. La possibilité demeure toujours que nos difficultés, non seulement avec ce problème de la relation entre. 
détermination et détermination primitive, mais aussi quant au sens de ce que Heidegger appelle avènement, manifestation de l'essence linguistique en tant que dire, proximité du rendre propre et du faire advenir, reçoivent un éclaircissement suffisant dans le corps de l'essai lui-même.

Aussi, ce que je propose de faire ici, sera simplement de dégager l'énoncé principal des conclusions de Heidegger sans ćlaborer les difficultés terminologiques que nous ne pouvions éviter de prendre en considération au début de l'essai. Une fois ces énonciations reconnues il nous sera possible de retourner au corps de l'essai pour y chercher la clarification désirée non seulement quant à ces énonciations, mais quant à leur vocabulaire.

En citant à nouveau le texte de Novalis, Heidegger attire l'attention sur ce qui détermine le caractère propre de l'essence linguistique, à savoir la manifestation de cette essence comme le dire, qui réside dans ce qu'il appelle la proximité du rendre propre et du faire advenir. Heidegger s'emploie maintenant à cerner ce caractère plus précisément. «Le langage, dit-il, seul est ce qui parle véritablement et il parle solitaire ${ }^{(265)}$. Ce point est à nouveau une répétition de ce que nous avons déjà noté dans les remarques d'introduction. Toutefois, ici, Heidegger établit la position ambiguë que le langage parle solitaire parce que le langage ne parle pas seul. L'important est que le langage ne parle pas dans l'isolement (seul) mais eu égard à ce qui appartient en même temps au parler du langage à savoir le parler de l'homme. Le langage parle solitaire en ce sens que le parler du langage est l'instance unifiante dans la relation entre le parler du langage et son articulation, mais les hommes parlent seulement dans la mesure où ils répètent ce qui est déjà entendu dans le parler du langage. Lorsque le caractère propre de la spécificité du langage est le dire, c'est le dire qui parle dans le parler du langage. Ainsi «l'homme ne peut parler que dans la mesure où, faisant partie du dire, il est à son écoute pour pouvoir, à la suite du dire, dire une parole ${ }^{(256)}$. Ainsi l'enjeu, semble-t-il, n'est pas seulement la relation entre le parler du langage et le parler des hommes, telle que nous l'avons observée dans les remarques d'introduction, mais tout à la. fois la 
parole que le dire prononce dans le parler du langage et la parole que l'homme énonce dans son propre parler sur le mode du dire-après. La difficulté est de comprendre plus clairement ce. que cette énonciation signifie.

La difficulté se redouble immédiatemment. Heidegger nous rappelle en effet que l'énonciation ne peut être exprimée dans une proposition. On pourrait alors s'attendre à ce que Heidegger, à défaut de poser une assertion, poursuive, comme dans l'introduction par la voie de la métaphore. Notre attente toutefois n'est pas remplie. Le caractère inexprimable de l'énonciation ne conduit pas ici au recours non-objectivant de la métaphore mais à la requête du silence. Mais ce sur quoi nous avons ici à garder le silence n'est pas l'énonciation elle-même, mais ce que Heidegger appelle, de manière embarrassante, «produire par le silence, dans l'être du langage, le mouvement qui fait advenir » (die ereignende Be-wëgung im Sprachwesen). Le néologisme de Be-wëgung n'est qu'un exemple parmi d'autres des nombreuses variations que Heidegger greffe sur la notion de mouvement (Bewegung) en association avec la notion de chemin (Weg). Le terme revient ici à une remarque antérieure: «le dire qui montre trace le chemin (be-wëgt) du langage au parler de l'homme ${ }^{(266)}$.

Il n'y a donc aucune équivoque dans l'expression «produire le mouvement qui fait advenir», puisque ce qui est en cause ici est de savoir comment l'énonciation communique le parler au parler de l'homme : autrement dit, de savoir comment l'énonciation confère la parole contenue dans le parler du langage au parler de l'homme. Puisque l'énonciation est inexprimable, Heidegger déclare ici qu'il nous faut garder le silence sur le mode de cette communication et, ajoute Heidegger, demeurer silencieux sur garder le silence. Ainsi écrit-il: «Le dire exige de nous que nous produisions par le silence (er-schweigen) dans l'être du langage le mouvement qui fait advenir, sans parler du silence ${ }^{(266)}$. La seule raison que Heidegger semble vouloir invoquer pour ce silence est que le mode de cette communication ne diffère pas de l'énonciation elle-même, car, écrit Heidegger, «le dire qui repose sur l'avènement est en tant que montrer le mode le plus propre à l'avènement ${ }^{(266)}$. L'avènement, faut-il. 
comprendre, parle et la manière de ce parler est l'énonciation. La manière dont l'avènement parle n'est pas toutefois «comme modus et espèce, mais la manière comme melos, le chant (das lied) qui dit en chantant ${ }^{(266)}$. L'avènement par conséquent, parle dans le chant du faire advenir qui énonce. Et ce dire qui fait advenir «porte ce qui est présent, à partir de ce qu'il a de propre, au paraître... ${ }^{(266)}$. La seule justification que donne Heidegger pour cette curieuse doctrine est un passage tiré de "Friedenfeier» où Hölderlin écrit que, puisque «Nous sommes un dialogue et à l'écoute les uns des autres», l'homme a expérimenté beaucoup...mais bientôt nous serons chant» ${ }^{(266)}$. L'ultime remarque de Heidegger sur la notion d'énonciation est faite en référence à la formule antérieure de la Lettre sur l'bumanisme, que «le langage est la maison de l'être». C'est bien en effet le cas puisque le langage en tant qu'énonciation est la manière (Weise) de l'avènement. «Le langage est la garde de la présence (Anwesen), dans la mesure où son paraître demeure confié (anvertraut) au montrer du dire qui fait advenir ${ }^{(267)}$.

5. Avant de tenter de reprendre cette doctrine sous forme de proposition, il est bon de décrire brièvement les quelques assertions importantes dans cette dernière partie de l'essai de Heidegger. En effet, outre les remarques sur le faire advenir de dire, Heidegger donne quelques indications sur la manière dont une telle doctrine doit être entendue.

La compréhension de cette doctrine fait problème parce que, comme on l'a vu, Heidegger revient sur sa déclaration initiale selon laquelle l'énonciation ne peut être représentée dans une proposition. Face à l'objection évidente que ses propres remarques sur l'énonciation sont elles-mêmes des propositions, il introduit ici une brève remarque sur ce qu'est une proposition: «Cela sonne comme un énoncé », dit-il, après avoir parlé sur le mode de l'événement. "Si nous le percevons sous cette forme, il ne donne rien à penser $\gg$ (zu-Denkende) ${ }^{(266)}$. Quant aux critères qui permettraient d'identifier ce qui est à penser et ce qui n'est pas à penser, Heidegger n'en souffle mot. Un peu plus haut, Heidegger remarque que nous ne pouvons pas comprendre ce que Novalis dit de la spécificité du langage en tant que déter- 
miné par l'avènement, si nous entendons par «compréhension» embrasser quelque chose dans la totalité de son essence. «Nous ne pouvons embrasser du regard l'être du langage parce que nous faisons nous-mêmes partie du dire ${ }^{(265)}$. Selon Heidegger, par conséquent, on ne peut embrasser du regard que ce en quoi on n'est pas soi-même impliqué essentiellement.

Mais dans la mesure où ce que nous embrassons du regard est l'avènement et où l'avènement advient dans l'énonciation et où l'énonciation humaine participe à l'énonciation de l'avènement, nous sommes nous-mêmes essentiellement impliqués dans ce que nous voudrions embrasser du regard.

En ce sens donc, nous ne pouvons par essence comprendre ce qui nous enveloppe par essence. Plus loin, Heidegger identifie cette notion de comprendre, en tant que voir quelque chose dans la totalité de son essence, avec ce qu'il appelle : «... le concept traditionnel du savoir défini à partir de la connaissance pensée comme représentation ${ }^{(266)}$. La thèse est donc que nous ne pouvons comprendre, représenter, embrasser du regard, dans la totalité de son essence et indépendamment de nous-même, l'énonciation - avènement, dans laquelle l'essence du langage comme langage devient manifeste.

Cette thèse repose sur deux suppositions fondamentales: que la compréhension est fondamentalement représentation et que l'essence de l'homme est impliquée au moins partiellement dans l'essence du langage. Que ces deux présuppositions soient discutables ne devrait pas nous empêcher de les considérer pour ce qu'elles sont. Heidegger conclut comme suit: «Parce que nous autres hommes, pour être ce que nous sommes, demeurons engagés dans l'être du langage et, par conséquent, ne pouvons jamais en sortir pour l'embrasser du regard à partir d'un autre lieu, nous n'apercevons jamais l'être du langage qu'autant que, quand luimême nous fait face, nous nous transportons en lui » ${ }^{(266)}$. Ainsi, nous ne saisissons l'essence du langage que si auparavant nous sommes appropriés par l'essence du langage. Cette doctrine est à rapprocher de la remarque antérieure selon laquelle «l'énonciation ne se laisse pas capter (einfangen) dans une proposition », à condition que nous admettions que le penser de la représentation 
caractéristique de la compréhension au sens de Heidegger, s'exprime dans des propositions.

Outre cette remarque sur la compréhension et la représentation par propositions, Heidegger formule à nouveau l'intention annoncée pour la première fois dans son introduction. Cette reformulation s'appuie sur l'importante remarque qui précède immédiatement, concernant la nécessité d'un changement de langage. En effet, le propos de Heidegger dans cet essai est de «préparer en quelque chose la transformation de notre rapport au langage »... Et il ajoute immédiatement : «L'expérience pourrait nai tre : toute pensée qui médite est un poématiser et tout poématiser est un penser ${ }^{(267)}$. Pensée méditante et poématisante vont donc de pair. Si une telle expérience pouvait naître, alors, estime Heidegger, notre relation au langage pourrait aussi prendre un nouveau cours.

Mais en quel sens notre relation au langage doit-elle se développer? Dans la mesure où nous ne pouvons ni comprendre ni représenter l'énonciation dans des propositions, notre réflexion sur l'essence du langage demeure problématique. Une telle réflexion, pose Heidegger, ne peut «être que «dire après le dire» (Nachsagen) ${ }^{(267)}$. Mais cette répétition rend nécessaire une nouvelle relation. Ce qu'il nous faut, ce ne sont donc ni de nouveaux mots, ni de nouvelles phrases, mais un changement dans notre relation même au langage. "Ce rapport se définit par la dispensation (Geschick) : si et comment nous sommes retenus par l'être du langage en tant qu'annonciation originelle de l'avènement en celui-ci ${ }^{(627)}$

Notre relation au langage se trouve déterminée parce que, selon Heidegger, "l'avènement est, comme ce qui est propre, ce qui porte en soi (eignend-haltend-ansichhaltend) un rapport de tous les rapports ${ }^{(266)}$. On n'a pas spécifié ce qu'est au juste notre relation au langage mais de quoi elle dépend. Selon l'intelligence de cette relation qui permettrait de donner un sens précis à ce que l'on a appelé la transformation de notre relation au langage, la seule chose que Heidegger ajoute est que «notre rapport au langage finit à partir de la manière dont en tant que tenu en mains (als Gebrauchten) nous appartenons à l'avènement » ${ }^{(267)}$. 
Tout est donc suspendu au sens donné aux notions d'annonciation originelle de l'avènement d'être du langage, ainsi qu'à la doctrine issue du rapport de tous les rapports. Peut-être trouvera-t-on quelque clarté dans le corps du texte, ce qui n'est pas ici le cas.

Du moins avons-nous appris que réfléchir dans l'essence du langage c'est répéter d'une certaine façon ce qui fait le propre de cette essence; c'est cette répétition qui requiert un changement de langage lui-même. Mais ce changement ne consiste pas à créer des mots nouveaux, ce n'est pas non plus une transformation que nous puissions «obtenir de force ${ }^{(267)}$. Toutefois, c'est un changement que nous pouvons, d'une certaine manière préparer.

La préparer serait réveiller l'expérience selon laquelle la pensée méditative et la poésie s'appartiennent mutuellement. C'est à préparer cette expérience que Heidegger s'emploie dans cet essai.

On ne nous a pas dit ce qu'est la relation entre l'essence de l'homme et l'essence du langage, ni en quoi consiste le changement dans cette relation. La seule chose que l'on ait dite est que ce changement dépend de certains facteurs obscurs, qu'elle ne peut être obtenue de force ni découverte, mais qu'elle peut être préparée par l'expérience de la co-appartenance de la pensée méditative et du poématiser. Si nous joignons ces remarques aux énoncés introductifs sur le propos et la méthode de cet essai, nous savons tout ce que l'on veut savoir concernant l'orientation fondamentale de l'essai.

6. Tentons maintenant d'élaborer les propositions de base qui régissent cette orientation. Puisque nous les avons lues à titre de remarques conclusives, nous y renvoyons comme à des propositions C: (C 1) La méditation sur l'essence du langage n'implique pas un changement de langage mais un changement dans notre relation au langage. (C 2) Cette relation est déterminée en accord avec notre propre langage. (C 3) Le changement dans cette relation peut être préparé par l'expérience de l'appartenance mutuelle du penser méditatif et du poématiser. (C 4) Cette préparation implique le projet de faire l'expérience à partir du langage lui-même du mouvement dynamique se développant en cercle à l'intérieur du 
langage lui-même. Ces propositions représentent le noyau initial, à quoi s'ajoute celui des déclarations conclusives : (C 5) Le langage seul est ce qui parle authentiquement et le langage parle solitaire. (C 6) Le caractère propre de l'essence du langage est déterminé par l'avènement. (C 7) Dans l'avènement réside la manifestation de l'essence du langage comme énonciation. (C 8) L'avènement parle dans la proximité de l'énonciation c'est-à-dire dans le chant de l'énonciation qui fait advenir. (C 9) L'énonciation fait parler la parole issue du parler du langage tandis que l'homme ne parle qu'en répétant la parole entendue dans ce parler. (C 10) L'énonciation ne peut être connue si par connaître on entend représenter ce qui se donne à penser. (C 11) L'homme doit garder le silence sur la manière dont le langage parle dans le parler humain et garder le silence sur son propre silence. (C 12) L'homme ne peut glisser un regard dans cette essence du langage quautant qu'il est déjà approprié par l'essence du langage.

Il faut bien avouer que cette liste de propositions est exposée au même genre de critiques que celles que l'on a extraites des remarques introductives. Toutefois, elle peut aider à saisir de manière grossière les étapes principales d'un itinéraire jalonné de difficultés. C'est donc moins un résumé qu'un ensemble de repères pour nous guider dans une pensée difficile et complexe.

7. Si l'on confronte les deux listes $\mathrm{I}$ et $\mathrm{C}$, on découvre certains recoupements importants : c'est le point de départ (I, 1, C 5), sur la notion d'essence du langage $(18,19, \mathrm{C} 6)$ sur la méthode (1 3 à 7, C 1 à 4), enfin l'évaluation (1 2, 1 10, C 10 à 11). Le point de départ et le point d'arrivée sont les mêmes: la citation de Novalis .Elle introduit l'idée générale que le langage n'a souci que de lui-même, en ce sens que le langage parle authentiquement dans la mesure où il parle et est solitaire.

Reste à comprendre ce que signifie ici authenticité, solitude, appliquées au parler du langage.

Les remarques sur l'essence du langage sont beaucoup plus complexes. Les expressions «le propre du langage » et «l'essence du langage comme langage » et «l'essence du langage» sont en gros équivalentes; on peut y joindre encore ce qui est dit du 
principe unifiant et du principe de différenciation qui sont inséparables, irréductibles et réciproques $(18,19)$. Au même thème se rattache l'idée d'une énonciation issue du parler du langage et entendue puis répétée dans le parler de l'homme (C 9). De même encore: le propre de l'essence du langage est déterminé par l'avènement $(C$ 6) dans lequel réside l'énonciation $(C$ 7) et qui est manifeste dans le chant où advient l'énonciation (C 8). Mais ces temarques accumulent les difficultés concernant le caractère propre et le caractère composé de cette essence, concernant le parler du langage et le parler de l'homme, enfin l'énonciation et la relation entre l'une et l'autre.

Les remarques sur la méthode sont également complexes. On peut admettre une équivalence approximative entre ce que l'introduction appelle «penser l'essence du langage comme tel » et ce que la conclusion appelle « réfléchir sur l'essence du langage $\gg$.

Quoiqu'inconnue, cette essence peut être tenue, à titre métaphorique, pour situer dans une région à laquelle conduit un chemin, que l'on peut suivre en raison de la relation entre l'essence humaine et l'essence du langage (1 3-4). Penser l'essence du langage c'est donc faire l'expérience de ce chemin du langage (1 5-6). Et penser, c'est ici percevoir le caractère propre de langage (1 7).

Enfin, ce penser enveloppe un changement dans notre rapport au langage qui peut être préparé par l'expérience de la mutuelle appartenance du penser méditatif et du poématiser (1 7, C 1-4). Si l'on approche tous ces termes proposés: connaissance représentative et indication métaphorique, perception et écoute, penser méditatif et poématiser, nous avons un réseau de relations qui constitue la notion centrale de l'expérience.

Enfin, les remarques sur l'évaluation portent à la fois sur l'essence du langage et sur l'énonciation. L'essence du langage, est-il dit, est inconnue ( 12 2). On ne peut la comprendre si par comprendre on entend la représentation dans les énoncés (C 10) et par énoncé des formulations verbales de ce qui est à penser (C 10), d'où la règle du silence (C 11). Un regard peut se glisser dans la mesure où l'homme est déjà approprié par l'essence du 
langage (C 12). Alors le caractère propre de l'être du langage peut être décrit conjecturalement.

Ces remarques impliquent une distinction entre connaître et jeter un regard, entre poser une assertion et conjecturer, entre formuler et faire silence. Ces distinctions elles aussi requièrent une détermination ultérieure.

Si maintenant nous gardons présentes à l'esprit ces deux séries de propositions, nous pouvons à nouveau lire l'introduction et la conclusion de Heidegger amenées à fusionner dans une série plus réglée de propositions les formulations issues de cette nouvelle lecture. On arrive alors à ceci :

1. Il y a une relation essentielle entre l'essence de l'homme et l'essence du langage parce que pouvoir parler est ce qui distingue l'homme en tant qu'homme.

2. Le parler de l'homme rend manifeste le parler du langage et le chant du langage rend manifeste la parole comme avènement.

3. L'essence du langage est unifiée et différenciée par un mouvement réfléchi sur lui-même dont les éléments sont inséparables, irréductibles et manifestes dans le parler du langage.

4. L'essence du langage est déterminée par l'avènement sur lequel repose l'énonciation.

5. Quoique l'essence du langage soit inconnue, elle peut être indiquée métaphoriquement comme ce qui repose dans une région vers laquelle un chemin est tracé.

6. Quoique l'essence du langage ne puisse être comprise au sens de embrasser dans une intention d'ensemble, on peut jeter sur elle un regard en écoutant le parler du langage.

7. Quoique l'essence du langage ne puisse être représentée dans des propositions, au sens de formation verbale de ce qui est à penser, on peut la décrire conjecturalement.

8. Réfléchir sur l'essence du langage au sens de répéter son caractère propre, implique que notre relation au langage soit transformée et que ce changement soit préparé par une ex- 
périence que nous ferions à partir du langage lui-même concernant l'appartenance mutuelle du penser méditatif et du poématiser.

9. C'est en s'engageant lui-même dans l'entrelacs du langage que l'homme peut faire l'expérience du mouvement réfléchi sur lui-même qui se déploie à l'intérieur même de l'essence du langage: alors l'homme réalise ce qui est indiqué par la formule du chemin ; il porte le langage en tant que le langage au langage.

La relecture du texte de Heidegger fait apparaître le défaut de clarification des propositions $1,2,7,8$ et les difficultés nouvelles qu'introduit l'essai de clarification dans les propositions 3, $4,5,6,7$. C'est ainsi que la première partie de l'essai de Heidegger juxtapose la notion classique du langage qu'on trouve dans le Peri Hermeneias d'Aristote avec la notion du langage issue de von Humboldt.

La seconde partie tente de suivre les indications suggérées par la formule du chemin dans le dessein de rendre compte de l'unité de l'essence du langage. La dernière partie, qui est aussi la plus importante, développe une méditation compliquée sur l'essence du langage comme énonciation. La manifestation de l'énonciation comme appropriation est la notion d'avènement ; au terme de l'entreprise, la formule initiale se précise: «porter le langage (l'être du langage) en tant que langage (énonciation) au langage (avec la parole articulée) ».

Tout repose finalement sur le défaut d'explication concernant la relation entre l'essence de l'homme et l'essence du langage et l'appel à une transformation de la relation de l'homme au langage.

Ce sont ces difficultés qui nous contraindront à chercher une lumière, du moins, dans les chapitres antérieurs d'Unterwegs zur Sprache.

Université d'Ottawa 